\title{
Calibrating the In-Medium Behavior of Quarkonia
}

\author{
Helmut Satz \\ Fakultät für Physik, Universität Bielefeld, D-33501 Bielefeld, Germany \\ Correspondence should be addressed to Helmut Satz; satz@physik.uni-bielefeld.de
}

Received 20 March 2013; Accepted 3 April 2013

Academic Editor: Jan E. Alam

Copyright (C) 2013 Helmut Satz. This is an open access article distributed under the Creative Commons Attribution License, which permits unrestricted use, distribution, and reproduction in any medium, provided the original work is properly cited.

\begin{abstract}
Quarkonium production has been considered as a tool to study the medium formed in high-energy nuclear collisions, assuming that the formation of a hot and dense environment modifies the production pattern observed in elementary collisions. The basic features measured there are the relative fractions of hidden to open heavy flavor and the relative fractions of the different hidden heavy flavor states. Hence the essential question is if and how these quantities are modified in nuclear collisions. We show how the relevant data must be calibrated; that is, what reference has to be used, in order to determine this in a model-independent way.
\end{abstract}

The original suggestion for charmonium production as a means to test the formation of a deconfined medium in highenergy nuclear collisions was based on the idea that color screening in such a medium would prevent the binding of charm quarks to a color neutral $J / \psi[1]$. The $J / \psi$ production process in elementary hadronic collisions (taking $p p$ as an example) begins with the formation of a $c \bar{c}$ pair; this pair can then either lead to open charm production (about 90\%) or subsequently bind to form a charmonium state (about 10\% for all charmonia). A schematic illustration (Figure 1) shows the dominant high-energy reaction through gluon fusion.

The initial $c \bar{c}$ production can be calculated in terms of the parton distribution functions $f_{p}$ of the relevant hadrons and the perturbative partonic cross-section. The full description of charmonium binding has so far resisted various theoretical attempts; on the other hand, the process is in good approximation independent of the incident hadronic collision energy $[2,3]$. This is a consequence of the fact that the heavy quark propagator in the reaction $g g \rightarrow c \bar{c}$ strongly dampens the mass variation of the $c \bar{c}$ pair with incident energy. Thus the fractions of the produced $c \bar{c}$ system into hidden versus open charm as well as those for the different charmonium states are approximately constant; once determined at one energy, they remain the same also for different energies. As a result, the phenomenological color evaporation model [4-7] provides a good description of charmonium production through the form

$$
\sigma_{h h \rightarrow J / \psi}(s)=g_{c \bar{c} \rightarrow J / \psi} \sigma_{h h \rightarrow c \bar{c}}(s),
$$

and correspondingly for the other charmonium states. Here the constant $g_{c \bar{c} \rightarrow J / \psi}$ specifies what fraction of the total $c \bar{c}$ production cross-section goes into $J / \psi$ production; in $p p$ collisions, it is typically about $2 \%$. The set of the different constants $g_{c \bar{c} \rightarrow i}$ for the different charmonium states $i$ thus effectively characterizes charmonium production in the absence of a medium.

A further important aspect of quarkonium production in elementary collisions is that the observed (1S) ground states $J / \psi$ and $\Upsilon$ are in both cases partially produced through feeddown from higher excited states [8-11]. Of the observed $J / \psi$ rates, only some $60 \%$ is a directly produced $J / \psi(1 S)$ state; about $30 \%$ comes from $\chi_{c}(1 \mathrm{P})$ and $10 \%$ from $\psi^{\prime}(2 \mathrm{~S})$ decay. Because of the narrow width of the excited states, their decay occurs well outside any interaction region.

The features we have here summarized for charm and charmonium production are readily extended to that of bottom and bottomonium. To simplify the discussion, we will continue referring to the charmonium case, keeping in mind that all arguments apply as well to bottomonia. Given the patterns observed in elementary collisions, we want to see how they are modified in the presence of a medium, as provided by nuclear collisions. From the point of view of production dynamics, one way such modifications can arise is as initial state effects, which take place before the $c \bar{c}$ pair is produced. The main possibilities considered so far are nuclear modifications of the parton distribution functions (shadowing or antishadowing) and a possible energy loss of 


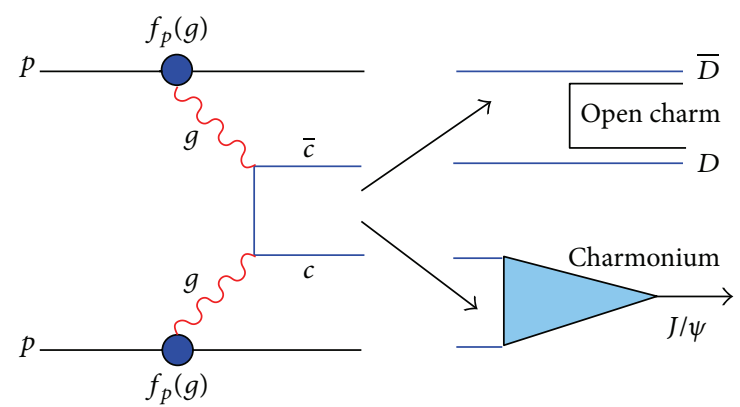

Figure 1: Schematic view of $J / \psi$ production in $p p$ collisions.

the partons passing through the nuclear medium to produce the $c \bar{c}$. Once produced, the pair can encounter final state effects, either in the form of a phase space shift already of the $c \bar{c}$, for example, through an energy loss of the unbound charm quarks, or through effects on the nascent or fully formed charmonium state. Such effects may arise from the passage through the cold nuclear medium or because of the presence of the medium newly produced in the nuclear collision. The latter is evidently what we have in mind when we want to use quarkonia to study quark-gluon plasma production. The difficulties encountered over the past years $[12,13]$ in arriving at a conclusive analysis of the relevant nuclear collision data on $J / \psi$ production are largely due to the problem of parametrizing the different effects and then constructing a convincing model correctly incorporating all of them. We want to show here that today experimental means have become available which allow us to calibrate the measured results in a way which avoids these difficulties.

Before we turn to the problem of determining medium effects on quarkonium production, we recall the two main conceptual approaches on what may happen; the two can perhaps best be labelled suppression and enhancement. Color screening in a quark-gluon plasma will decrease the quarkonium binding, both in strength and in its spatial range, and this should for sufficiently energetic nucleus-nucleus collisions lead to quarkonium dissociation or melting. Since the larger and less tightly bound states will melt at lower temperature or energy density than the ground states, color screening will produce sequential suppression $[14,15]$. We illustrate this for the $J / \psi$. After an initial threshold melting the $\psi^{\prime}$ and hence removing its feed-down component for $J / \psi$ production, there will be a second threshold for $\chi_{c}$ melting and then finally a third, at which the direct $J / \psi(1 S)$ is dissociated. The resulting pattern is illustrated in Figure 2. We have here introduced something denoted as $J / \psi$ survival probability. Theoretically, this is the chance of a $J / \psi$ to persist as a bound state in a deconfined medium. How to properly define this quantity as a useful observable in experimental studies is the subject of this paper.

The other alternative, $J / \psi$ enhancement, assumes, in accord with color screening and the resulting suppression, that at sufficiently high energies there is an almost complete dissociation of the $J / \psi$ 's produced in primary nucleonnucleon interactions. On the other hand, at such collision energies, these interactions lead to abundant $c \bar{c}$ production;

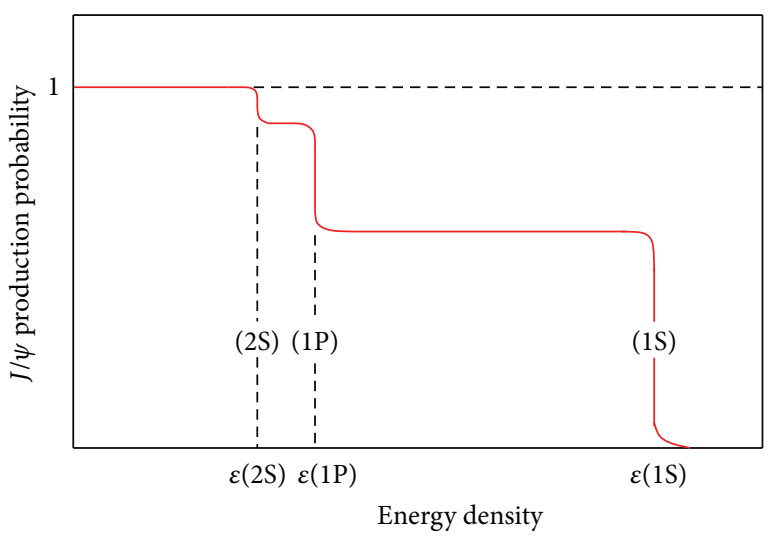

FIGURE 2: Schematic view of sequential $J / \psi$ suppression in a deconfined medium.

the rate for this process grows faster than that for the production of light quarks, and if the $c \bar{c}$ pairs remain present in the evolution of the medium, the system will at the hadronisation point show an oversaturation of charm, compared to the predicted thermal abundance. If these charm quarks have become part of an equilibrated medium and as such undergo hadronisation in the form of statistical combination, then such secondary charmonium formation can convert more $c \bar{c}$ pairs into $J / \psi$ 's than the dynamical primary production mechanism, thus leading to an effective $J / \psi$ enhancement [16-19], as shown in Figure 3. We note that this scenario invokes a new and so far unknown binding dynamics to form charmonia; the outcome is assumed to be determined simply by the relative abundance of charm quarks [20].

We now come to our central question: how to calibrate the $J / \psi$ survival? Since we are interested in using quarkonium production as a tool to study the medium produced in nuclear collisions, our primary concern is not if such collisions produce more or fewer $c \bar{c}$ pairs than proton-proton collisions, but rather if the presence of the medium modifies the fraction of produced $c \bar{c}$ pairs going into charmonium formation. In other words, the crucial quantity is the amount of charmonium production relative to that of open charm [21]. To illustrate, in $p p$ collisions, about $2 \%$ of the total $c \bar{c}$ production goes into $J / \psi$ 's. If in high-energy nuclear collisions the total $c \bar{c}$ production rate was reduced by a factor two, but we still have $2 \%$ of these going into $J / \psi$ 's, then evidently $A A$ collisions do not modify $J / \psi$ binding. This is, of course, strictly true only for fully integrated rates, which is difficult to check; we return to the issue of phase space variations shortly. But we note here already that in the example just given the number of $J / \psi$ 's produced in $A A$ collisions would be half of that obtained by scaling the results from $p p$ interactions. That is, however, not an indication of any $J / \psi$ suppression; it is just the consequence of having fewer $c \bar{c}$ pairs to start with, the formation probability of charmonia has remained the same as in $p p$ collisions, $2 \%$. It is thus crucial to correctly calibrate the survival in nuclear collisions.

To achieve that, we recall in both sequential suppression and statistical enhancement state that the total number of $c \bar{c}$ pairs produced in nuclear collisions is distributed among 


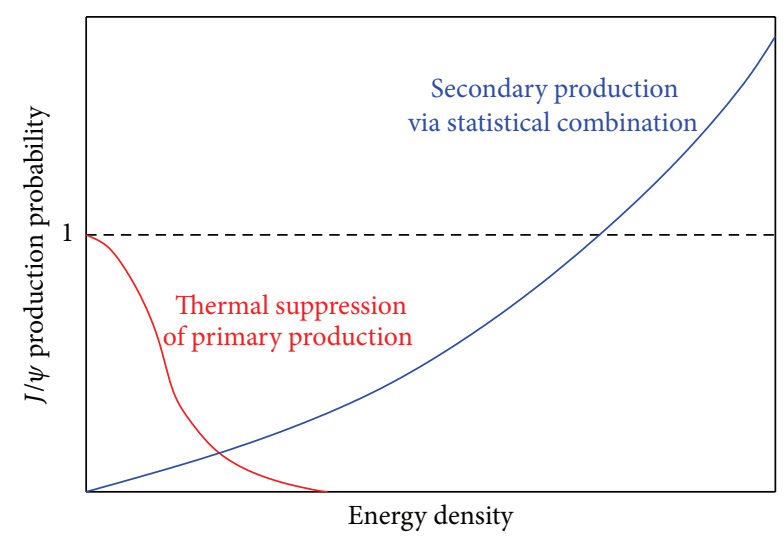

FIGURE 3: Schematic view of secondary production through statistical combination.

hidden and open charm differently than it is in protonproton collisions. The essential observable is thus the ratio of charmonium states to open charm in nuclear collisions, compared to that in $p p$ interactions. Sequential suppression predicts it to decrease with centrality and eventually vanish, statistical enhancement has it increasing with centrality, in many studies beyond the $p p$ value. Hence the relevant observable is the fraction of charmonia to open charm, or more generally, that of quarkonia to the relevant open heavy flavor production [21,22]. In this quantity, if measured over the entire phase space, the effects of possible initial state nuclear modifications-shadowing/antishadowing, parton energy loss-cancel out, so that whatever changes it shows relative to the $p p$ pattern are due to final state effects.

It should be noted here that in actual applications, it will most likely not be necessary (nor generally possible) to measure the total open charm production rate. The relative abundances of the different open charm states produced in high energy collisions are so far found to be in good agreement with the predictions of the statistical hadronisation model $[23,24]$, with a universal hadronisation temperature of some $150-170 \mathrm{MeV}$. For open charm production, this model assumes an initial dynamical (perturbative) $c \bar{c}$ production; subsequently, at the hadronisation point, the charm quarks thus produced form open charm hadrons according to their statistical abundances. The relative production rates are thus totally determined in terms of the mass of the open charm state and the universal hadronisation temperature. Such a description is found to hold very well for open charm production in high-energy interactions from $e^{+} e^{-}$ annihilation through $p p$ and $p A$ collisions; studies of the $A A$ behavior are underway. However, the model does not work for charmonium production, neither in elementary $\left(e^{+} e^{-}\right.$, $p p$ ) nor in nuclear interactions, since the binding mechanism forming charmonia is evidently of dynamical and not of statistical origin. In nuclear collisions, there are in addition medium effects on the binding.

As a consequence, we expect that the relative abundances of the different open charm states will not depend on the collision energy and remain essentially the same for elementary and nuclear collisions. Analogous to (1), the production rate $N\left(h h \rightarrow D_{i}\right)$ for a specific open charm state $D_{i}$ is then a constant fraction of the total open charm rate $N(h h \rightarrow c \bar{c})$ :

$$
N\left(h h \longrightarrow D_{i}\right) \simeq \bar{g}_{\bar{c} \bar{c} \rightarrow D_{i}} N(h h \longrightarrow c \bar{c}) .
$$

The crucial observable, the rate of $(J / \psi) /$ open charm, can thus in good approximation be taken as the rate of $(J / \psi) / D_{i}$ for a specific $D$-meson state. This will, of course, greatly facilitate the analysis.

The final state effect of interest to us is that caused by the newly produced medium. We, therefore, have to check to what extent the nascent or fully formed $J / \psi$ is already dissociated by the cold nuclear matter of the target or the projectile. This can be done by studying the ratio hidden to open charm in high-energy $p A$ collisions. It is in fact known that in such interactions there exist reductions of $J / \psi$ production beyond the scaled $p p$ results, but the origin of these is not unambiguously clarified. They could arise largely from initial state effects; but if there is a stronger suppression of $\psi^{\prime}$ than of $J / \psi$ at energies for which the state is formed inside the nuclear medium, this would indicate that there the interaction depends on its physical size. The different fates of the different quarkonium states in cold nuclear matter are due to their different sizes, in a hot medium due to the different binding energies. And dissociation by color screening should start with a threshold, while the breakup in nuclear matter is presumably continuous. Measurements of charmonia relative to open charm in $p A$ up to highest energies (RHIC, LHC) are, therefore, of great importance.

First applications of the in-medium charmonium study based on the relative survival of charmonia versus open charm were started last year, using LHC data from ALICE and CMS [25-28]. In Figure 4(a), we show mid-rapidity ALICE data for $J / \psi$ production at intermediate transverse momenta, compared to open charm production in a similar kinematic region. In Figure 4(b), the comparison is extended to larger transverse momenta, using CMS data for $J / \psi$ production. In both cases, $J / \psi$ production relative to $p p$ results, scaled by the number of collisions, decreases with increasing centrality, as seen by the corresponding $R_{A A}$ values.

This decrease has at times been considered as suppressed $J / \psi$ production. However, that is incorrect; the corresponding $R_{A A}$ for open charm production, as determined through $D$ measurements, shows within errors the same behavior. In other words, the reduction of the $J / \psi$ is in complete agreement with that of open charm; there is neither suppression nor enhancement; the fraction of the produced $c \bar{c}$ pairs going into $J / \psi$ production has remained the same in the $A A$ collisions which are considered here as in the corresponding $p p$ interactions:

$$
R_{A A}\left(\frac{J}{\psi}\right)=\frac{N_{A A}(J / \psi)}{n_{c} N_{p p}(J / \psi)}=\frac{N_{A A}(c \bar{c})}{n_{c} N_{p p}(c \bar{c})}=R_{A A}(c \bar{c})
$$

with $n_{c}$ denoting the scaling factor for the number of collisions at the corresponding centrality. We, therefore, have

$$
\frac{N_{A A}(J / \psi)}{N_{A A}(c \bar{c})}=\frac{N_{p p}(J / \psi)}{N_{p p}(c \bar{c})}=g_{c \bar{c} \rightarrow J / \psi}
$$




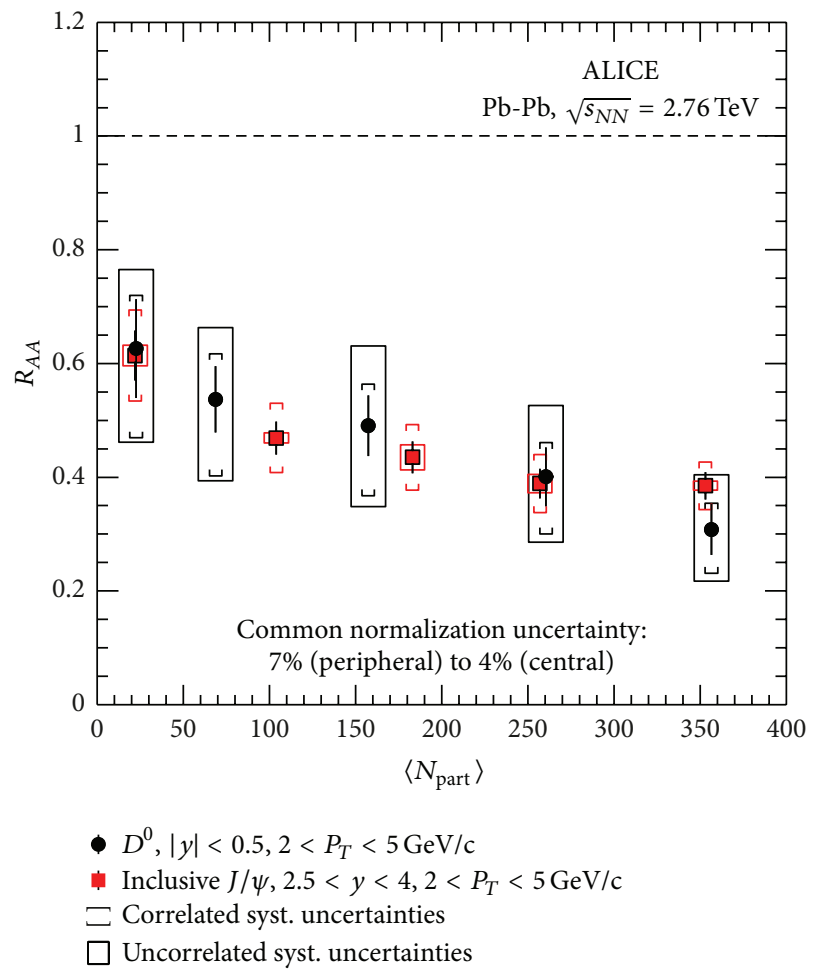

(a)

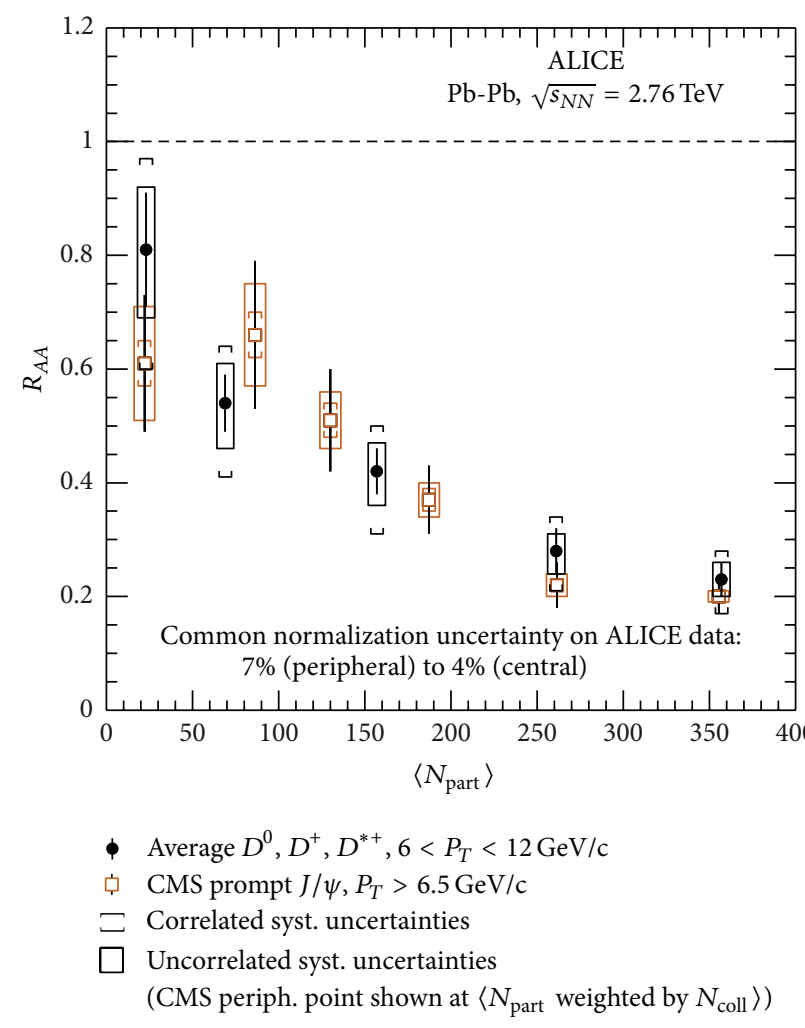

(b)

FIGURE 4: LHC data from ALICE and CMS [26-28], comparing $J / \psi$ production to open charm production at intermediate (a) and high (b) transverse momenta.

at all centralities, in the kinematic regime indicated. If we divide the $J / \psi$ rates by the open charm rates in the same kinematic region (large $P_{T}$ ), this ratio becomes centrality independent, and if we normalize it to the corresponding value from $p p$ collisions, it becomes unity. The correct and model-independent $J / \psi$ survival probability for experimental study is thus

$$
S_{J / \psi}=\frac{\left(N_{A A}(J / \psi) / N_{A A}(c \bar{c})\right)}{\left(N_{p p}(J / \psi) / N_{p p}(c \bar{c})\right)}=\frac{1}{g_{c \bar{c} \rightarrow J / \psi}}\left(\frac{N_{A A}(J / \psi)}{N_{A A}(c \bar{c})}\right) .
$$

In the kinematic regime considered so far, it is indeed unity, there is neither suppression nor enhancement.

At the LHC, corresponding data for low $P_{T}$ open charm production is not yet available. At RHIC, however, it is provided by the PHENIX and STAR collaborations [29-33], and the relevant comparisons of $J / \psi$ versus open charm production are shown in Figure 5. At high $P_{T}$ we have a similar behavior as at the LHC, no change of $J / \psi$ production relative to open charm. At low $P_{T}$, however, the $R_{A A}(c \bar{c})$ of open charm is within errors unity over the whole centrality range; in contrast, $R_{A A}(J / \psi)$ decreases strongly and thus here gives the correct $J / \psi$ survival probability. We see that now with increasing centrality, a smaller and smaller fraction of $c \bar{c}$ pairs go into $J / \psi$ production, with a suppression of up to $75 \%$ for the most central collisions. The $J / \psi$ production finally surviving could conceivably be large due to corona interactions [34].

In this context it seems of interest to note that a decrease of the open charm production rates between central and forward rapidity is observed in both $p A$ and $A A$ collisions relative to $p p$ collisions, at Fermilab $(p A)$ and at RHIC $(\mathrm{Cu}-$ $\mathrm{Cu})$. We note in particular in the Fermilab data based on $800 \mathrm{GeV} p A$ collisions (see Figure 6) that at forward rapidity there is a suppression of open charm production, relative to scaled $p p$ rates [35-38], while at midrapidity there is not. A similar effect is observed in $\mathrm{Cu}-\mathrm{Cu}$ collisions with $\sqrt{s}=$ $200 \mathrm{GeV}$ at RHIC [39]. If this is passed on to charmonium production, one expects for RHIC and LHC data a smaller $R_{A A}(J / \psi)$ at forward than at mid-rapidity, simply because there are fewer $c \bar{c}$ pairs there to form charmonia, no matter how. Such an effect was indeed observed at RHIC and has often been considered a puzzle [40].

The aim of this work was to show how quarkonium production has to be calibrated in order to obtain experimentbased, model-independent information on suppression or enhancement due to the hot medium formed in nuclear collisions. The data cited are given for illustration, to make the point. In a more complete study, the transverse momentum range of $J / \psi$ and open charm should be correlated more precisely. The crucial question now remains, of course, how 


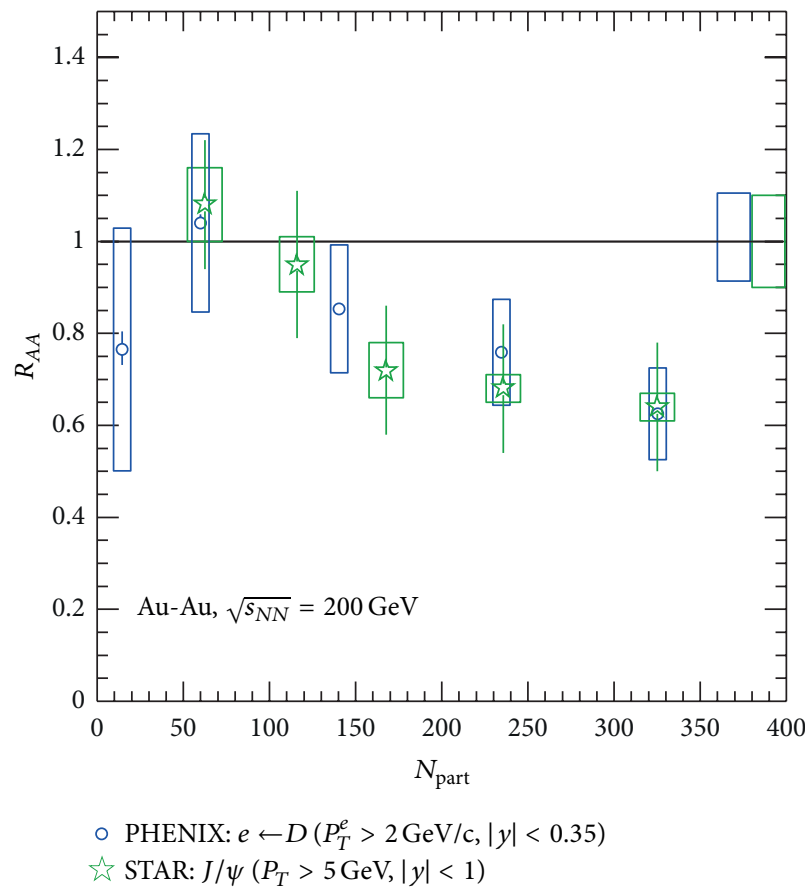

(a)

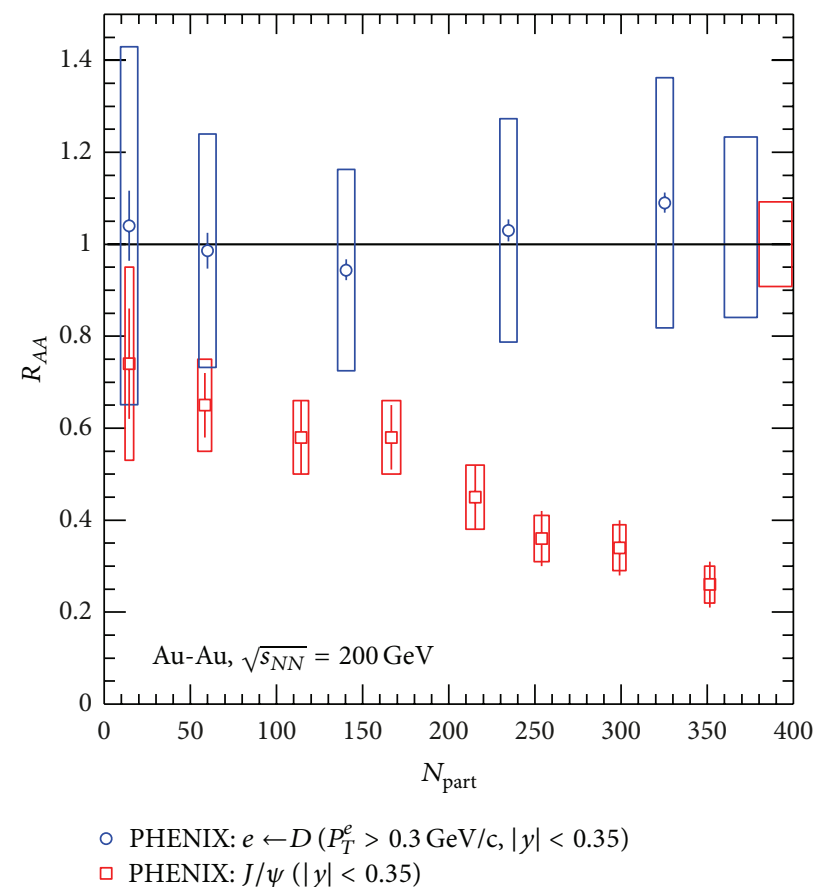

(b)

FIGURE 5: RHIC data from PHENIX and STAR [30-33], comparing $J / \psi$ production to open charm production at high (a) and low (b) transverse momenta.

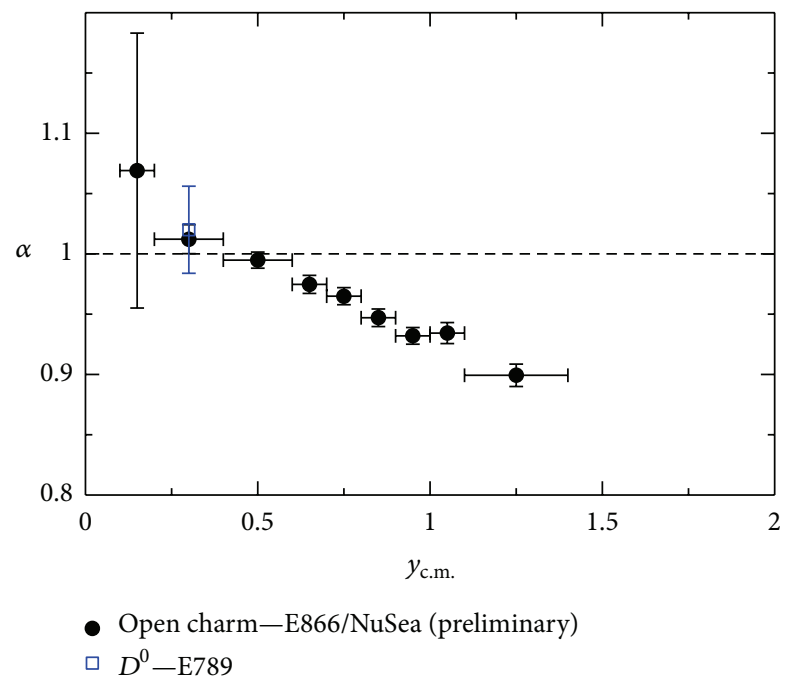

FIGURE 6: The rapidity dependence of open charm production in $p A$ collisions at $800 \mathrm{GeV}$, parametrized in the form $\sigma_{p A}=A^{\alpha} \sigma_{p p}$ [35-38].

the open charm rates for low $P_{T}$ production at the LHC will behave. That kinematic region is responsible for the bulk of $c \bar{c}$ production, and the issue to be decided is if nuclear collisions do or do not lead to an overall reduction. At RHIC, that is, not the case, as Figure 5(b) shows.

\section{Acknowledgments}

It is a pleasure for the author to thank Zaida Conesa del Valle, Torsten Dahms, Louis Kluberg, Mike Leitch, Carlos Lourenço, and Jürgen Schukraft for helpful discussions. Particular thanks go to Torsten Dahms also for pointing out the applicability of RHIC data for the analysis and for providing the author with the corresponding Figures.

\section{References}

[1] T. Matsui and H. Satz, " $J / \psi$ suppression by quark-gluon plasma formation," Physics Letters B, vol. 178, no. 4, pp. 416-422, 1986.

[2] R. Gavai, D. Kharzeev, H. Satz et al., "Quarkonium production in hadronic collisions," International Journal of Modern Physics A, vol. 10, pp. 3043-3070, 1995.

[3] R. Vogt, "Quarkonium baseline predictions," in Hard Probes in Heavy-Ion Collisions at the LHC, M. Mangano, H. Satz, and U. Wiedemann, Eds., CERN Yellow Report, 2004.

[4] M. B. Einhorn and S. D. Ellis, "Hadronic production of the new resonances: probing gluon distributions," Physical Review D, vol. 12, no. 7, pp. 2007-2014, 1975.

[5] H. Fritzsch, "Producing heavy quark flavors in hadronic collisions: a test of quantum chromodynamics," Physics Letters B, vol. 67 , no. 2, pp. 217-221, 1977.

[6] M. Glück, J. F. Owens, and E. Reya, "Gluon contribution to hadronic $J / \psi$ production," Physical Review D, vol. 17, pp. 23242331, 1978. 
[7] J. Babcock, D. Sivers, and S. Wolfram, "Quantumchromodynamic estimates for heavy-particle production," Physical Review D, vol. 18, no. 1, pp. 162-181, 1978.

[8] L. Antoniazzi, M. Arenton, Z. Cao et al., "Production of $J / \psi$ via $\psi^{\prime}$ and $\chi_{c}$ decay in $300 \mathrm{GeV} / \mathrm{c}$ proton- and $\pi \pm$-nucleon interactions," Physical Review Letters, vol. 70, no. 4, pp. 383-386, 1983.

[9] P. Faccioli, C. Lourenço, J. Seixas, H. K. Wöhri et al., "Study of $\psi^{\prime}$ and $\chi_{c}$ decays as feed-down sources of $J / \psi$ hadro-production," Journal of High Energy Physics, vol. 2008, no. 10, pp. 1-15, 2008.

[10] A. Adare, S. Afanasiev, C. Aidala et al., "Ground and excited state charmonium production in $p+p$ collisions at $\sqrt{s}=$ 200 GeV," Physical Review D, vol. 85, p. 092004, 2012.

[11] N. Brambilla, M. Kramer, R. Mussa et al., "Heavy quarkonium physics," CERN Yellow Report, Quarkonium Working Group, 2005.

[12] H. Satz, "Colour deconfinement and quarkonium binding," Journal of Physics G, vol. 32, no. 3, pp. R25-R69, 2006.

[13] L. Kluberg and H. Satz, "Color deconfinement and charmonium production in nuclear Collisions," in Landolt-Börnstein Set 2010: Relativistic Heavy Ion Physics, R. Stock, Ed., Springer, 2010.

[14] F. Karsch and H. Satz, "The spectral analysis of strongly interacting matter," Zeitschrift für Physik C, vol. 51, no. 2, pp. 209-224, 1991.

[15] F. Karsch, D. Kharzeev, and H. Satz, "Sequential charmonium dissociation," Physics Letters B, vol. 637, no. 1-2, pp. 75-80, 2006.

[16] P. Braun-Munzinger and J. Stachel, "On charm production near the phase boundary," Nuclear Physics A, vol. 690, no. 1-3, pp. 119-126, 2001.

[17] R. L. Thews, M. Schroedter, and J. Rafelski, "Enhanced $J / \psi$ production in deconfined quark matter," Physical Review $C$, vol. 63, no. 5, p. 054905, 2001.

[18] L. Grandchamp and R. Rapp, "Charmonium suppression and regeneration from SPS to RHIC," Nuclear Physics A, vol. 709, no. 1-4, pp. 415-439, 2002.

[19] R. L. Thews and M. L. Mangano, "Momentum spectra of charmonium produced in a quark-gluon plasma," Physical Review C, vol. 73, no. 1, p. 014904, 2006.

[20] P. Braun-Munziger and J. Stachel, "Charmonium from statistical hadronization of heavy quarks - a probe for deconfinement in the quark-gluon plasma," in Landolt-Börnstein Set 2010: Relativistic Heavy Ion Physics, R. Stock, Ed., Springer, 2010.

[21] K. Sridhar and H. Satz, "Charmonium production versus open charm in nuclear collisions," Physical Review D, vol. 50, no. 5, pp. 3557-3559, 1994.

[22] A. Andronic, P. Braun-Munziger, and K. Redlich, "Statistical hadronization of heavy quarks in ultra-relativistic nucleusnucleus collisions," Nuclear Physics A, vol. 789, no. 1-4, pp. 334356, 2007.

[23] F. Becattini, P. Castorina, J. Manninen, and H. Satz, "The thermal production of strange and non-strange hadrons in $\mathrm{e}^{+} \mathrm{e}^{-}$ collisions," The European Physical Journal C, vol. 56, no. 4, pp. 493-510, 2008.

[24] A. Andronic, F. Beutler, P. Braun-Munzinger, K. Redlich, and J. Stachel, "Statistical hadronization of heavy flavor quarks in elementary collisions: successes and failures," Physics Letters B, vol. 678, no. 4, pp. 350-354, 2009.

[25] Z. Conesa del Valle, Quark Matter 2012, Washington and private communication.

[26] B. Abelev, J. Adam, D. Adamová et al., "Suppression of high transverse momentum $\mathrm{D}$ mesons in central $\mathrm{Pb}-\mathrm{Pb}$ collisions at
$\sqrt{s_{\mathrm{NN}}}=2.76$, Journal of High Energy Physics, vol. 2012, no. 9, pp. 1-37, 2012.

[27] B. Abelev, J. Adam, D. Adamová et al., " $J / \psi$ suppression at forward rapidity in $\mathrm{Pb}-\mathrm{Pb}$ collisions at $\sqrt{s_{\mathrm{NN}}}=2.76 \mathrm{TeV}$," Physical Review Letters, vol. 109, no. 7, p. 072301, 2012.

[28] S. Chatrchyan, V. Khachatryan, A. M. Sirunyan et al., "Suppression of non-prompt $J / \psi$, prompt $J / \psi$, and $\Upsilon(1 \mathrm{~S})$ in $\mathrm{PbPb}$ collisions at $\sqrt{s_{\mathrm{NN}}}=2.76 \mathrm{TeV}$," Journal of High Energy Physics, vol. 2012, no. 5, pp. 1-54, 2012.

[29] T. Dahms, private communication.

[30] A. Adare, S. Afanasiev, C. Aidala et al., "Heavy-quark production in $p+p$ and energy loss and flow of heavy quarks in $\mathrm{Au}+\mathrm{Au}$ collisions at $\sqrt{s_{\mathrm{NN}}}=200 \mathrm{GeV}$,' Physical Review C, vol. 84, no. 4, p. 044905,2011 .

[31] A. Adare, S. Afanasiev, C. Aidala et al., " $J / \psi$ production versus centrality, transverse momentum, and rapidity in $\mathrm{Au}+\mathrm{Au}$ collisions at $\sqrt{s_{\mathrm{NN}}}=200 \mathrm{GeV}$," Physical Review Letters, vol. 98, no. 23, p. 232301, 2007.

[32] F. Akram and M. A. K. Lodhi, " $B_{c}$ absorption cross sections by $\rho$ mesons," Physical Review C, vol. 84, no. 6, p. 064912, 2011.

[33] L. Adamczyk, G. Agakishiev, M. M. Aggarwal et al., " $J / \psi$ production at high transverse momenta in $p+p$ and $\mathrm{Au}+\mathrm{Au}$ collisions at $\sqrt{s_{\mathrm{NN}}}=200 \mathrm{GeV}$,' Physics Letters B, vol. 722, no. 1-3, pp. 55-62, 2013, arXiv:1208.2736 [nucl-ex].

[34] S. Digal, H. Satz, and R. Vogt, "Charmonium production and corona effect," Physical Review C, vol. 85, no. 3, p. 034906, 2012.

[35] M. Leitch, private communication.

[36] M. J. Leitch, J. Boissevain, T. A. Carey et al., "Nuclear dependence of neutral D production by $800 \mathrm{GeV} / \mathrm{c}$ protons," Physical Review Letters, vol. 72, p. 2542, 1994.

[37] M. J. Leitch and J. C. Peng, E866/NuSea, private communication.

[38] S. Klinksiek, Ph. D. thesis, The University of New Mexico, 2005.

[39] N. Apadula, in Proceedings of the 29th Winter Workshop on Nuclear Dynamics, Squaw Valley, Calif, USA, February 2013.

[40] R. Granier de Cassagnac, "Quarkonia production in cold and hot matters," Journal of Physics G, vol. 35, no. 10, p. 104023, 2008. 

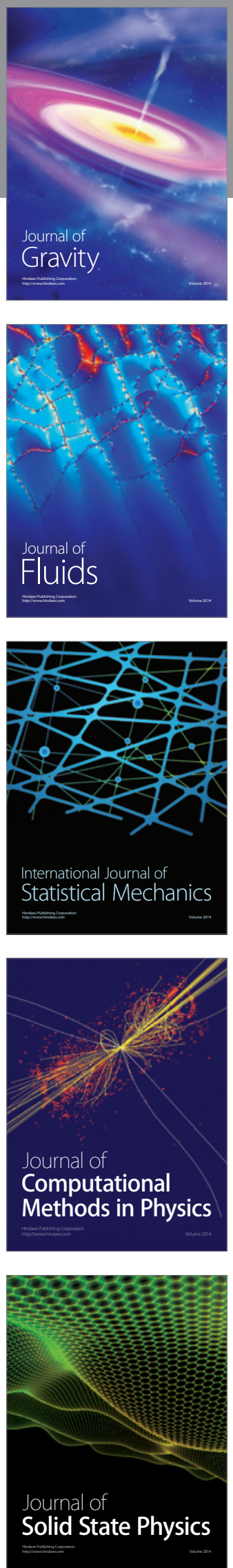

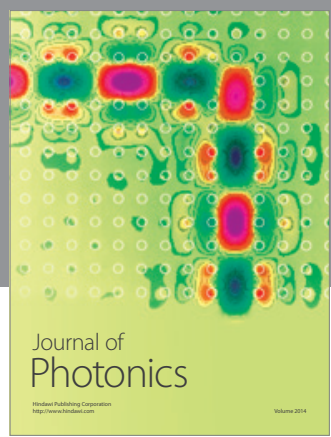

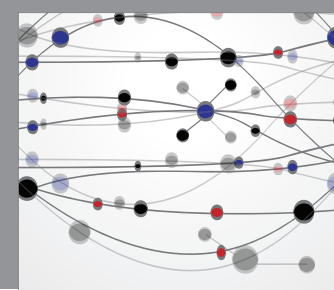

The Scientific World Journal

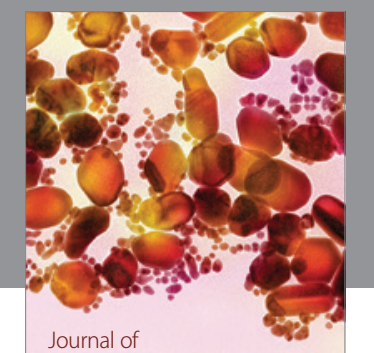

Soft Matter
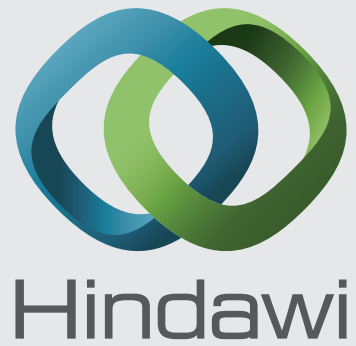

Submit your manuscripts at

http://www.hindawi.com
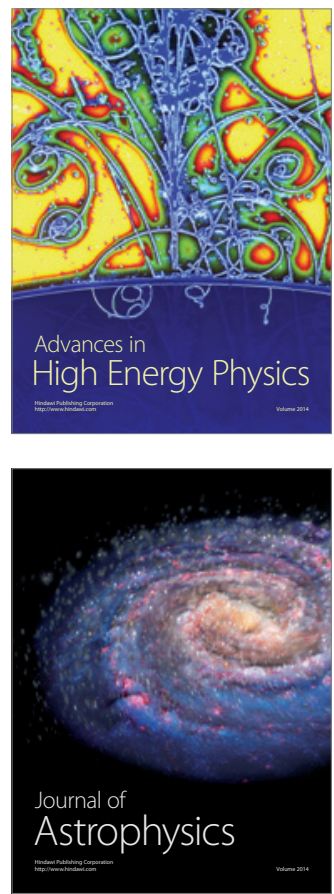
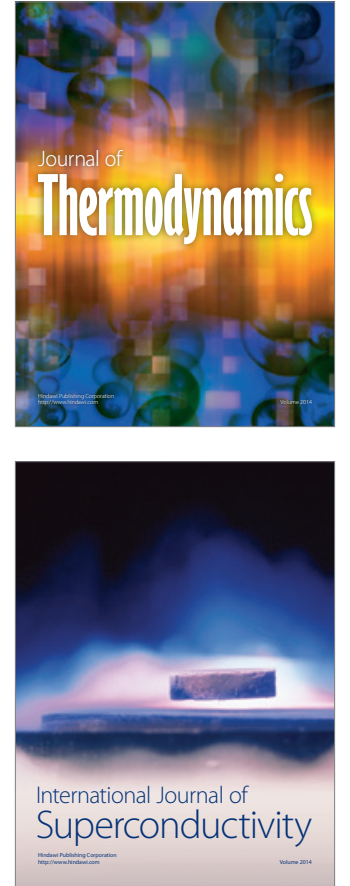
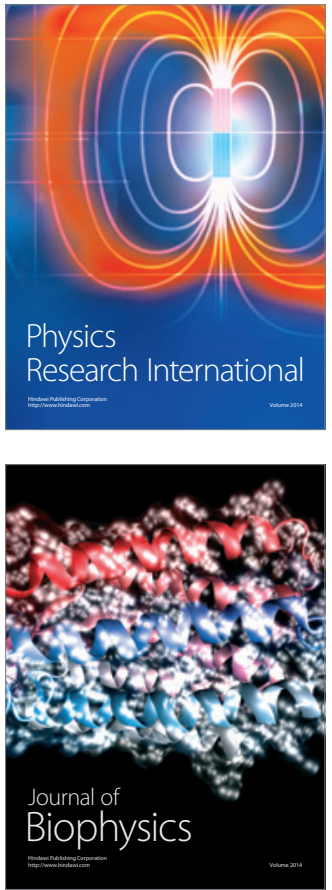
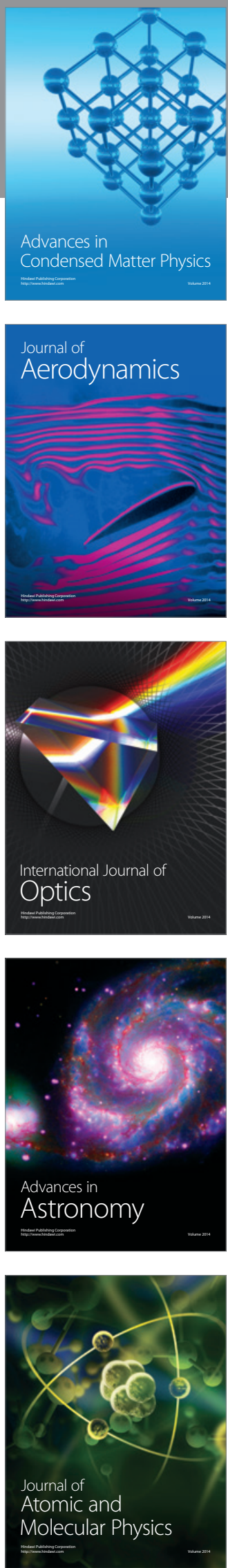